\section{social
research}

- The term 'pilot studies' refers to mini versions of a full-scale study (also called 'feasibility' studies), as well as the specific pre-testing of a particular research instrument such as a questionnaire or interview schedule.
- Pilot studies are a crucial element of a good study design. Conducting a pilot study does not guarantee success in the main study, but it does increase the likelihood.
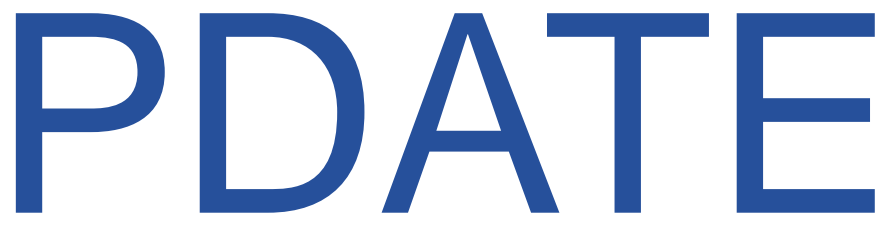

- Pilot studies fulfil a range of important functions and can provide valuable insights for other researchers. There is a need for more discussion amongst researchers of both the process and outcomes of pilot studies.

\title{
The importance of pilot studies
}

\section{Edwin R. van Teijlingen and Vanora Hundley}

The term pilot study is used in two different ways in social science research. It can refer to so-called feasibility studies which are "small scale version [s], or trial run[s], done in preparation for the major study" (Polit et al., 2001: 467). However, a pilot study can also be the pre-testing or 'trying out' of a particular research instrument (Baker 1994: 182-3). One of the advantages of conducting a pilot study is that it might give advance warning about where the main research project could fail, where research protocols may not be followed, or whether proposed methods or instruments are inappropriate or too complicated. In the words of De Vaus (1993: 54) "Do not take the risk. Pilot test first." These are important reasons for undertaking a pilot study, but there are additional reasons, for example convincing funding bodies that your research proposal for the main study is worth funding. Thus pilot studies are conducted for a range of different reasons (see Table 1)

Pilot studies can be based on quantitative and/or qualitative methods and large-scale studies might employ a number of pilot studies before the main survey is conducted. Thus researchers may start with "qualitative data collection and analysis on a relatively unexplored topic, using the results to design a subsequent quantitative phase of the study" (Tashakkori \& Teddlie 1998: 47). The first phase of a pilot might involve using in-depth interviews or focus groups to establish the issues to be addressed in a large-scale ques- tionnaire survey. Next the questionnaire, e.g. the wording and the order of the questions, or the range of answers on multiple-choice questions, might be piloted. A final pilot could be conducted to test the research process, e.g. the different ways of distributing and collecting the questionnaires. For example, a recent study exploring nurses' and midwives' attitudes to research followed this pattern. In this study focus groups were used to identify key issues from which a questionnaire could be developed, and this was then piloted prior to the study proper (Hundley et al. 2000). On a much larger scale, the largest (decennial) survey in the UK, the Census (of 29 ${ }^{\text {th }}$ April 2001), tested methodological and other changes to the $1991 \mathrm{Cen}$ sus questionnaire on over 100,000 households in 1997. This 1997 Census Test "provided essential information on public reaction to new questions and form style as well as assessing the success of collection and processing methods" (Office for National Statistics, General Register Office for Scotland, Northern Ireland Statistical \& Research Agency 1999: 15).

Pilot studies may also try to identify potential practical problems in following the research procedure. For example, in a recent Scottish study of maternity care the pilot phase demonstrated that the proposed means of distributing the questionnaires would not be adhered to (van Teijlingen $e t$ al. 2001). Without consulting the research team, the person responsible for distribut-
Dr Edwin van Teijlingen is a medical sociologist and Senior Lecturer in Public Health at the Department of Public Health, University of Aberdeen. His research interests include the organisation of maternity care at home and abroad, substance misuse, and psychosocial aspects of genetics.

Dr Vanora Hundley is a midwife and Lecturer at the Centre for Advanced Studies in Nursing, University of Aberdeen. Her research interests include midwifery care, consumer satisfaction and preferences, and research utilisation. 


\section{social research UPDATE}

ing the questionnaires from the hospital records department decided that it was better to distribute them through the community midwives. This was despite the fact that the hospital itself had suggested the records department as a means of distribution. Other problems such as poor recording and response rates can also be identified and precautionary procedures or safety nets can be devised.

The steps used to pilot a questionnaire on a small group of volunteers, who are as similar as possible to the target population, are listed in Table 2.

Pilot studies can also uncover local politics or problems that may affect the research process. In the study described above, the managers of maternity services had different perceptions of what the forthcoming changes in the Data Protection Act (1998) allowed them to do about the involvement of their clients in research. In the above mentioned recent Scottish study of maternity care one head of midwifery voiced ethical concerns about the use of follow-up or reminder letters due to a previous local incident, where parents of an ill baby had been sent a questionnaire which was felt to be inappropriate, and as a result of changes to the UK Data Protection Act. Consequently reminders were sent out via the head of midwifery in case there were any problems with the newborn baby.

\section{Problems of pilot studies}

It should be recognised pilot studies may also have a number of limitations. These include the possibility of making inaccurate predictions or assumptions on the basis of pilot data; problems arising from contamination; and problems related to funding. These issues are now discussed in turn.

Completing a pilot study successfully is not a guarantee of the success of the full-scale survey. Although pilot study findings may offer some indication of the likely size of the response rate in the main survey, they cannot guarantee this because they do not have a statistical foundation and are nearly always based on small numbers. Furthermore, other problems or headaches may not be- come obvious until the larger scale study is conducted.

A further concern is that of contamination. This may arise in two ways:

1. where data from the pilot study are included in the main results;

2. where pilot participants are included in the main study, but new data are collected from these people.

Social scientists engaged in predominantly quantitative research are likely to argue that: "an essential feature of a pilot study is that the data are not used to test a hypothesis or included with data from the actual study when the results are reported" (Peat et al. 2002: 57). The obvious concern is that if there were problems with the research tool and modifications had to be made in the light of the findings from the pilot study, data could be flawed or inaccurate. However, where an established and validated tool is being used and the pilot study is determining other methodological aspects such as recruitment rates, it could be argued that such data may be of value.

A common problem isthe inclusion of pilot study participants in the site(s) of the main study. Here the concern is that such participants have already been exposed to an intervention and, therefore, may respond differently from those who have not previously experienced it. This may be positive, for example the participants may become more adept at using a new tool or procedure. However it may also be negative with participants showing a decline in following a protocol because it is no longer novel. Indeed both changes in behaviour have long been recognised and a 'run in' period, where an intervention is introduced prior to a study, is often used for these reasons. The concern about including participants from the pilot study in the main study arises because only those involved in the pilot, and not the whole group, will have had the experience. In some cases however it is simply not possible to exclude these pilot-study participants because to do so would result in too small a sample in the main study. This problem arises in particular where the samples are clusters, for example schools, prisons or hospitals. In such cases one can conduct a
Table 1. Reasons for conducting pilot studies

- Developing and testing adequacy of research instruments

- Assessing the feasibility of a (fullscale) study/survey

- Designing a research protocol

- Assessing whether the research protocol is realistic and workable

- Establishing whether the sampling frame and technique are effective

- Assessing the likely success of proposed recruitment approaches

- Identifying logistical problems which might occur using proposed methods

- Estimating variability in outcomes to help determining sample size

- Collecting preliminary data

- Determining what resources (finance, staff) are needed for a planned study

- Assessing the proposed data analysis techniques to uncover potential problems

- Developing a research question and research plan

- Training a researcher in as many elements of the research process as possible

- Convincing funding bodies that the research team is competent and knowledgeable

- Convincing funding bodies that the main study is feasible and worth funding

- Convincing other stakeholders that the main study is worth supporting 


\section{social research UPDATE}

sensitivity analysis (or sub-group analysis) to assess to what extent the process of piloting influences the size of the intervention effect.

Contamination is less of a concern in qualitative research, where researchers often use some or all of their pilot data as part of the main study. Qualitative data collection and analysis is often progressive, in that a second or subsequent interview in a series should be 'better' than the previous one as the interviewer may have gained insights from previous interviews which are used to improve interview schedules and specific questions. Some have therefore argued that in qualitative approaches separate pilot studies are not necessary (e.g. Holloway 1997:121). For example, a qualitative interviewer conducting 15 focus group interviews will listen to the recordings or read through the transcripts of the first three or four in order to improve the questions, the way of introducing the issues into the group interview or even to add new topics. Thus, although there is no specific pilot study, analysis of the earlier focus groups may help improve the later ones. However, Frankland and Bloor (1999: 154) argue that piloting provides the qualitative researcher with a "clear definition of the focus of the study" which in turn helps the researcher to concentrate data collection on a narrow spectrum of projected analytical topics. Piloting of qualitative approaches can also be carried out if "the researcher lacks confidence or is a novice, particularly when using the interview technique" (Holloway 1997: 121). Problems may also arise where a pilot study requires a significant investment of resources, making it difficult for the study team to call a halt to the research after an unsuccessful pilot study. Researchers might be tempted to make considerable changes in the main study, rather than deciding that the proposed study is not possible with the available resources, time, population, etc. In contrast, funding bodies may be reluctant to fund a further study if the pilot has been substantial as they may view the research as no longer original, especially if results from the pilot study are published.

\section{Why are pilot studies not reported?}

Publication bias may occur because of a tendency for journals to accept only papers that have statistically significant results and not to report non-significant effects (Mahoney 1977; Chann 1982; Dickersin, 1990). A recent study exploring research on passive smoking found a difference of two years in the median time to publication between findings from significant and non-significant studies (Misakian \& Bero 1998). It follows that papers reporting methodological issues, such as those identified during the pilot phase of a study, will also be less attractive to publishers.

Selective publication of research results has been recognised as a problem. It may lead to an overestimation of the effectiveness of interventions, exposing patients to useless or harmful treatments, while overestimation of adverse effects may mean that patients are denied effective forms of care (Oxman et al. 1994). In the past editors have recognised the dangers of publication bias with respect to clinical trials and have offered 'an amnesty for unpublished trials' in an attempt to overcome these problems (Smith \& Roberts, 1997). However, it is equally important to ensure that lessons learned with respect to the research method are shared, otherwise patients may be subjected to poorly developed tools or money may be wasted because methods of recruitment failed. A consistent selection bias favouring reports of primary research over papers on research methods, theoretical thinking, or secondary analysis, can lead to many of researchers re-inventing the wheel without having had the opportunity to learn from other people's experience.

\section{Conclusions}

It has been said that pilot studies are likely to be "underdiscussed, underused and underreported" (Prescott and Soeken, 1989 p60). Full reports of pilot studies are rare in the research literature (Lindquist, 1991; Muoio et al, 1995, van Teijlingen et al. 2001). When reported, they often only justify the research methods or particular research tool used. Too often research papers only refer to one element of the pilot study, for exam-
Table 2. Pilot study procedures to improve the internal validity of a questionnaire

- administer the questionnaire to pilot subjects in exactly the same way as it will be administered in the main study

- ask the subjects for feedback to identify ambiguities and difficult questions

- record the time taken to complete the questionnaire and decide whether it is reasonable

- discard all unnecessary, difficult or ambiguous questions

- assess whether each question gives an adequate range of responses

- establish that replies can be interpreted in terms of the information that is required

- check that all questions are answered

- re-word or re-scale any questions that are not answered as expected

- shorten, revise and, if possible, pilot again

(Source: Table 3.23 in Peat et al. 2002: 123)

social research UPDATE is distributed without charge on request to social researchers in the United Kingdom by the Department of Sociology at the University of Surrey as part of its commitment to supporting social research training and development.

Contributions to social research UPDATE that review current issues in social research and methodology in about 2,500 words are welcome. All UPDATE articles are peer-reviewed. 


\section{social research UPDATE}

ple, to the 'pre-testing' or 'pilot testing' of a questionnaire (De Vaus, 1993). Such papers simply state: "the questionnaire was tested for validity and reliability." When pilot studies are mentioned in more detail in academic papers and reports, researchers regularly comment that they "had learned from the pilot study" and made the necessary changes, without offering the reader details about what exactly was learnt. Some of these processes and outcomes from both successful and failed pilot studies might be very useful to others embarking on projects using similar methods and instruments. This is particularly important because pilot studies can be "time-consuming, frustrating, and fraught with unanticipated problems, but it is better to ... deal with them before investing a great deal of time, money, and effort in the full study" (Mason and Zuercher, 1998). It has also been argued that the current research climate demands accountability from researchers, which means that there is a need to ensure the best possible use of research results (Crosswaite and Curtice 1994). We would like to go one step further and argue that researchers have an ethical obligation to make the best use of their research experience by reporting issues arising from all parts of a study, including the pilot phase.

Well-designed and well-conducted pilot studies can inform us about the best research process and occasionally about likely outcomes. Therefore investigators should be encouraged to report their pilot studies, and in particular to report in more detail the actual improvements made to the study design and the research process.

\section{References}

Baker, T.L. (1994), Doing Social research (2 ${ }^{\text {nd }}$ Edn.), New York: McGraw-Hill Inc.

Bowling, A. (1997), Research Methods in Health: Investigating Health \& Health Services, Buckingham: Open University Press. p 232

Burns, N. and Grove, S.K. (1999), Understanding Nursing Research (2 ${ }^{\text {nd }}$ edn.), Philadelphia: W.B. Saunders Company. p 40.

Crombie, I.K. and Davies, H.T.O. (1997), Research in Health Care: Design, Conduct and Interpretations of Health Services Research, London: Wiley.
Crosswaite, C. and Curtice, L. (1994), Disseminating research results-the challenge of bridging the gap between health research and health action. Health Promotion International 9: 289.

De Vaus, D.A. (1993), Surveys in Social Research ( $3^{\text {rd }}$ edn.), London: UCL Press.

Frankland, J. and Bloor, M. (1999), Some issues arising in the systematic analysis of focus group material, In: Barbour, R. and Kitzinger, J. (eds) Developing Focus Group Research: Politics, Theory \& Practice, London: Sage

Holloway, I. (1997). Basic Concepts for Qualitative Research, Oxford: Blackwell Science.

Hundley, V, Milne, J, Leighton-Beck, L. et al. (2000). Raising research awareness among midwives and nurses: does it work? Journal of Advanced Nursing 31 (1): 78-88.

Lindquist, R. (1991). Don't forget the pilot work! Heart Lung 20: 91-92

Mason, D.J. and Zuercher, S.L. (1995). Pilot studies in clinical nursing research. Journal of the New York State Nursing Association 26: 11 .

Muoio, R., Wolcott, L., and Seigel, H. (1995). A win-win situation: The pilot program. Journal of Continuing Education in Nursing. 26: 230-233.

Office for National Statistics, General Register Office for Scotland, Northern Ireland Statistical \& Research Agency. (1999), 2001 Census Information Paper, Government Statistical Services: London.

Peat, J., Mellis, C., Williams, K. and Xuan W. (2002), Health Science Research: A Handbook of Quantitative Methods, London: Sage.

Polit, D.F., Beck, C.T. and Hungler, B.P. (2001), Essentials of Nursing Research: Methods, Appraisal and Utilization. 5th Ed., Philadelphia: Lippincott Williams \& Wilkins

Prescott, P.A. and Soeken, K.L. (1989), The potential uses of pilot work. Nursing Research 38: 60-62.

Rosenberg, K.M. and Daly, H.B. (1993), Foundations of Behavioural Research: A Basic Question Approach, Fort Worth/ London: Harcourt Brace College Publishers. p 182-184.

Tashakkori, A and Teddlie, C. (1998), Mixed Methodology: Combining Qualitative \& Quantitative Approaches, Sage
Teijlingen van, E., Rennie, A.M., Hundley, V., Graham, W. (2001), The importance of conducting and reporting pilot studies: the example of the Scottish Births Survey, Journal of Advanced Nursing 34: 289-295 social research UPDATE (ISSN: 1360-7898)

is published by

Department of Sociology University of Surrey Guildford GU2 5XH United Kingdom.

Tel: 01483300800

Fax: 01483689551

Edited by Nige/ Gillbert

(e-mail: gng@soc.surrey.ac.uk) 